\title{
Scalar curvature and projective embeddings, II
}

\author{
S. K. Donaldson \\ February 1, 2008
}

\section{Introduction}

This is a sequel to the previous paper [6], which studied connections between the differential geometry of complex projective varieties and certain specific "balanced" embeddings in projective space. The original plan was that this sequel would be a lengthy paper, discussing various extensions and ramifications of the ideas sudied in [6]. However this plan has been modified in the light of subsequent developments. On the one hand, Mabuchi [1], [12], [13. has extended the results of [6] to the case where the varieties have infinitesimal automorphims. On the other hand, Phong and Sturm [14, 15] have sharpened some of the arguments in [6]. They also explain the relation of the ideas to the Deligne pairing and the Chow norm, and to earlier work of Zhang [18, which the author was unfortunately not aware of when writing [6]. These developments mean that some of the results planned for the sequel are now redundant, while on the other hand the exposition of all the different points of view has grown into a daunting task. Thus, instead, this sequel is a short paper devoted to the proof of one result which is quite an easy consequence of the main theorem in [6].

To state our result, suppose that $X$ is a compact Kahler manifold and fix a Kahler class $\left[\omega_{0}\right.$ ] on $X$. Recall that the Mabuchi functional [9] is a functional $\mathcal{M}$, defined up to an arbitrary additive constant, on the Kahler metrics in this cohomology class which is characterised by the formula

$$
\delta \mathcal{M}=\int_{X}(S-\hat{S}) \delta \phi \frac{\omega^{n}}{n !}
$$

Here metrics $\omega$ in the class are represented by Kahler potentials $\omega=\omega_{0}+i \bar{\partial} \partial \phi$ and $\delta \phi$ is an infinitesimal variation in $\phi$. The symbol $S$ denotes the scalar curvature of the metric $\omega$ and $\hat{S}$ is the average value of $S$ with respect to the volume form $\frac{\omega^{n}}{n !}$, which is a topological invariant of the Kahler class. What 
equation (1) really defines is a 1-form on the space of metrics in the Kahler class and one shows that this is closed, so is the derivative of a function $\mathcal{M}$, unique up to a constant.

Now suppose that $L$ is a positive line bundle over $X$ and the Kahler class is $2 \pi c_{1}(L)$. As in [6], we write $\operatorname{Aut}(X, L)$ for the group of automorphims of the pair $(X, L)$ modulo the trivial automorphisms $\mathbf{C}^{*}$ (acting by constant scalar multiplication on the fibres).

Theorem 1 Suppose that $\operatorname{Aut}(X, L)$ is discrete and that there is a metric $\omega_{\infty}$ of constant scalar curvature in the Kahler class $2 \pi c_{1}(L)$. Then $\omega_{\infty}$ minimises the Mabuchi functional in this Kahler class.

No doubt the hypothesis on the automorphism group can be relaxed, using the techniques of [1]. In the case of Kahler-Einstein metrics this result was proved by Bando and Mabuchi in [1].

Before beginning the proof we include some general discussion. It is clear from the definition that a constant scalar curvature metric $\omega_{\infty}$ is a critical point of $\mathcal{M}$ and it is also easy to see that the it is a local minimiser. Thus the difficulty in proving the theorem is to go from this local picture to the space of all Kahler metrics. In fact it is more convenient to work with the space $\mathcal{K}$ of Kahler potentials The main theme of the proof is the notion of convexity, for the functional $\mathcal{M}$ and various related functionals. This is related to the uniqueness of the constant scalar curvature metric which was the main result of [6]. The notion of convexity depends on a suitable geometric structure on the space $\mathcal{K}$. One obvious structure is realised by the embedding of $\mathcal{K}$ as an open set in the vector space $C^{\infty}(X)$, and convexity has a meaning in this sense. However, as far as the author knows, there is no reason to think that $\mathcal{M}$ is convex in this obvious sense. A more subtle geometric structure on $\mathcal{K}$ arises from considerations of symplectic geometry, yielding a Riemannian metric making $\mathcal{K}$ formally into a symmetric space, dual to a group $\mathcal{G}$ of symplectomorphisms of $X$ (or, more precisely, the extension of the group of exact symplectomorphisms by $\left.S^{1}\right)([10$, , 17, [5]). Convexity has a meaning in this sense, as convexity along the geodesics in $\mathcal{K}$, and indeed $\mathcal{M}$ is convex from this point of view. Moreover this convexity property arises as part of a general package of results, related to the fact that the scalar curvature furnishes a moment map for an action of the symplectomorphism group. As explained in [5], if one knew that any two points in $\mathcal{K}$ can be joined by a geodesic segment then Theorem 1 (without any restrictive hypotheses) would be an immediate consequence of this convexity. The construction of geodesic segments in $\mathcal{K}$ is a very difficult analytical problem but great progress in developing this approach has been made by X.X.Chen. While it is perhaps 
not likely that all points can in fact be joined by smooth geodesics, in [2] Chen proved an existence theorem for $C^{1,1}$ geodesics which enabled him to deduce the bound on the Mabuchi functional in the case when $\hat{S}$ is negative. Chen's approach has been extended in recent work of Chen and Tian 3], who prove a more general result, subsuming our Theorem 1. However we hope that the proof here will still have some interest.

Our proof of Theorem 1 develops the approach of $[6$. There are two main parts of the proof. In the first part (Section 2) we consider another functional on the space $\mathcal{K}$ which can be related to a finite-dimensional problem on the symmetric space $M=G L(d, \mathbf{C}) / U(d)$. The main result here is Theorem 2 below. In the second part (Section 3 ) we replace the line bundle $L$ by $L^{k}$ for large $k$ and apply the main result of [ 6 , and the asymptotic theory used there. In the first part of the we emphasise the symmetry in the discussion between the spaces $M$ and $\mathcal{K}$. All of this can be fitted in to the general picture of [6], which started from commuting actions of the two groups $U(d)$ and $\mathcal{G}$. With the exception of Lemma 4, the picture we develop in Section 2 largely follows from these group actions and general constructions concerning symplectic quotients. However in order to prove Lemma 4 we need to have the relevant formulae written down explicitly, and all-in-all we make a fresh start, so that this paper can be read independently of [6]. The proof of Lemma 4 hinges on the convexity of another functional $I$ on $\mathcal{K}$ in the first, more obvious, sense.

\section{2}

Let $X, L$ be as above and suppose that we have a fixed subspace $E \subset$ $H^{0}(X ; L)$, of dimension $d$, which gives a projective embedding of $X$. Let $h$ be a Hermitian metric on the line bundle $L \rightarrow X$. This defines a unitary connection on $L$, with curvature $-i \omega_{h}$ say, and we have

$$
\omega_{e^{\phi} h}=\omega_{h}+i \bar{\partial} \partial \phi
$$

Let $\mathcal{K}$ denote the set of metrics $h$ such that $\omega_{h}$ is a positive $(1,1)$ form on $X$. Thus if we fix a base point $h_{0}$ in $\mathcal{K}$ we can identify this space with the set of Kahler potentials,

$$
\mathcal{K} \cong\left\{\phi: \omega_{h_{0}}+i \bar{\partial} \partial \phi>0\right\}
$$

We write

$$
d \mu_{h}=\frac{\omega_{h}^{n}}{n !}:
$$


the standard volume form associated to the Kahler metric $\omega_{h}$, and we write $V$ for the volume of $X$, computed using any of these metrics.

Now let $M$ denote the set of Hermitian metrics on the finite-dimensional vector space $E$. We have two fundamental constructions:

- Given $h \in \mathcal{K}$ we get a metric $\operatorname{Hilb}(h) \in M$, the standard $L^{2}$-metric defined using the fibre metric on $L$ and the volume form $d \mu_{h}$ but with a convenient normalising factor

$$
\|s\|_{\operatorname{Hilb}(h)}^{2}=\frac{d}{V} \int|s|_{h}^{2} d \mu_{h} .
$$

So we have a map

$$
\text { Hilb }: \mathcal{K} \rightarrow M
$$

- Given $H \in M$ we define a metric $F S(H)$ on $L$ by decreeing that if $s_{\alpha}$ is an orthonormal basis of $E$ with respect to $H$ then

$$
\sum_{\alpha}\left|s_{\alpha}\right|_{F S(H)}^{2}=1
$$

pointwise on $X$. The corresponding form $\omega_{F S(H)}$ is the restriction of the standard Fubini-Study metric on $\mathbf{P}\left(E^{*}\right)$ to the image of $X$ under the projective embedding, so it is a positive form and we have a map

$$
F S: M \rightarrow \mathcal{K}
$$

A "balanced metric", in the sense of [6], corresponds to a pair $\left(h^{*}, H^{*}\right)$ with

$$
\operatorname{Hilb}\left(h^{*}\right)=H^{*}, F S\left(H^{*}\right)=h^{*},
$$

that is, to a fixed point of either composite $F S \circ$ Hilb or Hilb $\circ F S$.

Suppose we fix a non-zero element $\Theta \in \Lambda^{\operatorname{dim} E} E$. Then we can define the determinant of any metric $H$ in $M$. (This is just the determinant of the matrix of $H$ with respect to any basis $\left(s_{\alpha}\right)$ of $E$ with $\left.\Theta=s_{1} \wedge \ldots \wedge s_{d}\right)$. Thus we have a map

$$
\log \text { det }: M \rightarrow \mathbf{R} \text {. }
$$

A different choice of $\Theta$ just changes this map by the addition of a constant. We need the analogous map on $\mathcal{K}$. We recall that there is a functional $I: \mathcal{K} \rightarrow \mathbf{R}$, unique up to an additive constant, characterised by the condition that if $h_{t}=e^{\phi_{t}} h_{0}$ is a path in $\mathcal{K}$ then

$$
\frac{d I}{d t}=\int_{X} \dot{\phi} d \mu_{h_{t}}
$$


This functional has been used by many authors. As for the Mabuchi functional, essentially what is defined by equation (5) is a 1 -form on $\mathcal{K}$, and one has to check that this is 1 -form is closed. Notice that the derivative of the volume form is given by

$$
\left(\frac{d}{d t}\right) d \mu_{h_{t}}=\Delta_{t}^{\prime}(\dot{\phi}) d \mu_{h_{t}}
$$

where $\Delta_{t}^{\prime}$ denotes one half the ordinary Riemannian Laplacian of the metric $\omega_{h_{t}}$. (We use the sign convention in which the Laplacian is a nonegative operator.) This gives

$$
\frac{d^{2} I}{d t^{2}}=\int_{X}\left(\ddot{\phi}+\dot{\phi} \Delta^{\prime} \dot{\phi}\right) d \mu_{h_{t}}
$$

Lemma 1 For any $h, h_{0} \in \mathcal{K}$, with $h=e^{\phi} h_{0}$ we have

$$
\int_{X} \phi d \mu_{h_{0}} \leq I(h)-I\left(h_{0}\right) \leq \int_{X} \phi d \mu_{h}
$$

This is also well-known, and amounts to the fact that $I$ is a convex function on $\mathcal{K}$, regarded as an open subset of $C^{\infty}(X)$. By symmetry it suffices to prove the left-hand inequality. We write $h_{t}=e^{t \phi} h_{0}$ and consider the function

$$
f(t)=\int_{X} t \phi d \mu_{h_{0}}-\left(I\left(h_{t}\right)-I\left(h_{0}\right)\right)
$$

on $[0,1]$. Both $f(0)$ and $f^{\prime}(0)$ vanish and

$$
f^{\prime \prime}(t)=-\int_{X} \phi \Delta_{t}^{\prime} \phi d \mu_{h_{t}} \leq 0
$$

so $f(1) \leq 0$.

Clearly we have the following scaling identities, for $\alpha \in \mathbf{R}$,

$$
\begin{aligned}
\operatorname{Hilb}\left(e^{\alpha} h\right) & =e^{\alpha} \operatorname{Hilb}(h), \quad F S\left(e^{\alpha} H\right)=e^{\alpha} F S(H), \\
\log \operatorname{det}\left(e^{\alpha} H\right) & =\log \operatorname{det} H+\alpha d, \quad I\left(e^{\alpha} h\right)=I(h)+\alpha V .
\end{aligned}
$$

We now define maps

$$
\begin{aligned}
\mathcal{L} & =\log \operatorname{det} \circ \text { Hilb }: \mathcal{K} \rightarrow \mathbf{R} \\
Z & =-I \circ F S: M \rightarrow \mathbf{R}
\end{aligned}
$$


We also put

$$
\tilde{\mathcal{L}}=\mathcal{L}-\frac{d}{V} I, \tilde{Z}=Z+\frac{V}{d} \log \operatorname{det}
$$

so $\tilde{\mathcal{L}}$ and $\tilde{Z}$ are unchanged by constant scaling on $\mathcal{K}$ and $M$ respectively.

Given a metric $h \in \mathcal{K}$, we define a function $\rho_{h}$ on $X$ by

$$
\rho_{h}=\frac{d}{V} \sum_{1}^{d}\left|s_{\alpha}\right|_{h}^{2},
$$

where $\left(s_{\alpha}\right)$ is an orthonormal basis for $E$ with respect to $\operatorname{Hilb}(h)$. The function $\rho_{h}$ does not depend on the choice of this orthonormal basis.

Lemma 2 The derivative of $\mathcal{L}$ on $\mathcal{K}$ is given by

$$
\delta \mathcal{L}=\int_{X}\left(\Delta^{\prime} \rho_{h}+\rho_{h}\right)(\delta \phi)
$$

To see this note first that changing the element $\Theta$ changes $\mathcal{L}$ by a constant and does not affect the derivative of $\mathcal{L}$. So to verify the formula at a given point $h_{0} \in \mathcal{K}$ we may suppose that there is an orthonormal basis $\left(s_{\alpha}\right)$ with respect to $\operatorname{Hilb}\left(h_{0}\right)$ such that $s_{1} \wedge \ldots s_{d}=\Theta$. For any $\phi$ we have $\mathcal{L}\left(e^{\phi} h_{0}\right)=\log \operatorname{det} H_{\phi}$ where $H_{\phi}$ is the matrix with entries $\left\langle s_{\alpha}, s_{\beta}\right\rangle_{\operatorname{Hilb}\left(e^{\phi} h_{0}\right)}$. Differentiating at the given point $\phi=0$, where $H_{\phi}$ is the identity matrix, we have

$$
\delta \mathcal{L}=\sum_{\alpha} \delta\left\|s_{\alpha}\right\|_{\operatorname{Hilb}\left(e^{\phi} h_{0}\right)}^{2} .
$$

Now, pointwise on $X$,

$$
\delta\left|s_{\alpha}\right|_{e^{\phi} h_{0}}^{2}=(\delta \phi)\left|s_{\alpha}\right|_{h_{0}}^{2}, .
$$

When we take the $L^{2}$ norm over $X$ we get another term from the variation in the volume form (3); thus

$$
\delta\left\|s_{\alpha}\right\|_{\operatorname{Hilb}(h)}^{2}=\frac{d}{V} \int_{X}\left(\delta \phi+\Delta^{\prime}(\delta \phi)\right)\left|s_{\alpha}\right|_{h_{0}}^{2} d \mu_{h_{0}} .
$$

Summing over $\alpha$ and integating by parts we obtain

$$
\begin{aligned}
\sum_{\alpha} \delta\left\|s_{\alpha}\right\|_{\operatorname{Hilb}(h)}^{2} & =\int_{X}\left(\delta \phi+\Delta^{\prime}(\delta \phi)\right) \rho_{h_{0}} d \mu_{h_{0}} \\
& =\int_{X}\left(\rho_{h_{0}}+\Delta^{\prime} \rho_{h_{0}}\right) \delta \phi d \mu_{h_{0}},
\end{aligned}
$$

as required. 
Corollary $1 A$ point $h^{*}$ in $\mathcal{K}$ is balanced if and only if it is a critical point of the functional $\tilde{\mathcal{L}}=\mathcal{L}-\frac{d}{V} I$ on $\mathcal{K}$.

This is just because $\delta \mathcal{L}$ vanishes for all $\delta \phi$ of integral zero if and only if $\Delta^{\prime} \rho_{h}+\rho_{h}$ is a constant, but this can only occur if $\rho_{h}$ is a constant, since $\Delta^{\prime}$ is a non-negative operator. The factor inserted in the definition of Hilb then implies that $h=F S(H i l b(h))$.

Symmetrically we have

Lemma 3 The derivative of $Z$ at a point $H$ of $M$ is given by

$$
\delta Z=\sum_{\alpha \beta} \int_{X}(\delta H)_{\alpha \beta}\left(s_{\alpha}, s_{\beta}\right)_{F S(H)} d \mu_{F S(H)},
$$

where $\left(s_{\alpha}\right)$ is an orthonormal basis of $E$ with respect to $H$.

The proof is completely straightforward. It follows then that a balanced point (regarded as fixed point of Hilb $\circ F S$ on $M$ ) is the same as a critical point of the function $\tilde{Z}=Z+\frac{V}{d} \log \operatorname{det}$ on $M$.

We can now state the main result of this section,

Theorem 2 Suppose that there is a balanced point $h$ in $\mathcal{K}$. Then $h$ is an absolute minimum of the functional $\tilde{\mathcal{L}}$ on $\mathcal{K}$.

To prove this we consider the function $P$ on the product $\mathcal{K} \times M$ given by

$$
P(h, H)=\log \operatorname{Tr}\left(\operatorname{Hilb}(h) H^{-1}\right) .
$$

In other words

$$
P(h, H)=\log \sum_{\alpha}\left\|s_{\alpha}\right\|_{\mathrm{Hilb}(h)}^{2},
$$

where $\left(s_{\alpha}\right)$ is an $H$-orthonormal basis of $E$. From the definitions, we have

$$
P(h, \operatorname{Hilb}(h))=\log d, P(F S(H), H)=\log d,
$$

for all $h \in \mathcal{K}, H \in M$.

We put

$$
\tilde{P}(h, H)=P(h, H)-\log d+\frac{1}{d} \log \operatorname{det} H-\frac{1}{V} I(h),
$$

then

$$
\tilde{P}(h, \operatorname{Hilb}(h))=\frac{1}{d} \tilde{\mathcal{L}}(h), \quad \tilde{P}(F S(H), H)=\frac{1}{V} \tilde{Z}(H) .
$$

We now turn to the finite-dimensional side, with the function $Z$ on $M$, the set of Hermitian metrics on $E$. There is a standard metric on $M$, regarded as a symmetric space $G L(d, \mathbf{C}) / U(d)$ and the geodesics in this metric are just the images of the 1-parameter subgroups in $G L(d, \mathbf{C})$. The crucial result we need is 
Proposition 1 The function $Z$ is convex along geodesics in $M$.

This result is essentially equivalent to that of Zhang [18, and is also implicit in [6]. In the framework of [6] this is an instance of the convex function one always obtains associated to a Hamiltonian action of a group on a Kahler manifold (see [7, Chapter 6, for example). This is the well-known "KempfNess principle" 8]. However we give a direct proof here since it is quite short (once one knows the relevant identities to use).

A general geodesic in $M$ can be written as

$$
H_{t}=\operatorname{diag}\left(e^{\lambda_{\alpha} t}\right)
$$

with respect to some basis $s_{\alpha}$, orthonormal with respect to $H_{0}$. By definition

$$
Z\left(H_{t}\right)=-I\left(h_{t}\right),
$$

where $h_{t}=F S\left(H_{t}\right)$. Thus applying $(7)$, and evaluating at $t=0$,

$$
\frac{d^{2} Z}{d t^{2}}=-\int_{X} \dot{\phi} \Delta^{\prime} \dot{\phi}+\ddot{\phi} d \mu_{h_{0}} .
$$

Now the sections

$$
\exp \left(-\frac{\lambda_{\alpha}}{2} t\right) s_{\alpha}
$$

are orthonormal with respect to $H_{t}$ so by definition $h_{t}=e^{\phi_{t}} h_{0}$ where

$$
\phi_{t}=-\log \sum_{\alpha} e^{-\lambda_{\alpha} t}\left|s_{\alpha}\right|_{h_{0}}^{2} .
$$

Then, using the fact that $\sum_{\alpha}\left|s_{\alpha}\right|_{h_{0}}^{2}=1$ we have, at $t=0$,

$$
\begin{gathered}
\dot{\phi}=\sum \lambda_{\alpha}\left|s_{\alpha}\right|^{2}, \\
\ddot{\phi}=-\sum \lambda_{\alpha}^{2}\left|s_{\alpha}\right|^{2}+\left(\sum \lambda_{\alpha}\left|s_{\alpha}\right|^{2}\right)^{2} .
\end{gathered}
$$

We have then

$$
\ddot{Z}=\int_{X}-\frac{1}{2}|\nabla \dot{\phi}|^{2}+\sum_{\alpha} \lambda_{\alpha}^{2}\left|s_{\alpha}\right|_{h_{0}}^{2}-\left(\sum_{\alpha} \lambda_{\alpha}\left|s_{\alpha}\right|_{h_{0}}^{2}\right)^{2} .
$$

We denote by $($, ) any of the four natural $\mathbf{R}$-bilinear pairings

$$
\begin{array}{rll}
T^{*} X & \times\left(T^{*} X \otimes L\right) \rightarrow L & L \times\left(T^{*} X \otimes L\right) \rightarrow T^{*} X \\
L & \times L \rightarrow \mathbf{R} & T^{*} X \times T^{*} X \rightarrow \mathbf{R}
\end{array}
$$


obtained using the metrics on $L$ and $T^{*} X$ furnished by $h_{0}$. The crucial identity we need is that for any function $f$ on $X$,

$$
|\nabla f|^{2}=2 \sum_{\alpha}\left|\left(\nabla f, \nabla s_{\alpha}\right)\right|_{h_{0}}^{2}
$$

This follows, with a little thought, from the definition of the metric $F S\left(H_{0}\right)$ the restriction of the Fubini-Study metric, see [6] page 502, but note that there is an error there in the factor of 2 above. Consider the positive function

$$
F=\sum_{\alpha}\left|\left(\nabla \dot{\phi}, \nabla s_{\alpha}\right)-\left(\lambda_{\alpha}-\dot{\phi}\right) s_{\alpha}\right|_{h_{0}}^{2}
$$

on $X$. Expanding out, and applying (16) with $f=\dot{\phi}$,

$$
F=\frac{1}{2}|\nabla \dot{\phi}|^{2}+\sum_{\alpha}\left(\lambda_{\alpha}-\dot{\phi}\right)^{2}\left|s_{\alpha}\right|_{h_{0}}^{2}-2\left(\left(\nabla \dot{\phi}, \nabla s_{\alpha}\right), s_{\alpha}\right)\left(\lambda_{\alpha}-\dot{\phi}\right) .
$$

Now we have an identity

$$
\left(\left(\nabla \dot{\phi}, \nabla s_{\alpha}\right), s_{\alpha}\right)=\left(\nabla \dot{\phi},\left(s_{\alpha}, \nabla s_{\alpha}\right)\right)
$$

and $\nabla\left|s_{\alpha}\right|_{h_{0}}^{2}=2\left(s_{\alpha}, \nabla s_{\alpha}\right)$ so

$$
2\left(\left(\nabla \dot{\phi}, \nabla s_{\alpha}\right), s_{\alpha}\right)\left(\lambda_{\alpha}-\dot{\phi}\right)=\left(\nabla \dot{\phi}, \nabla\left|s_{\alpha}\right|_{h_{0}}^{2}\right)\left(\lambda_{\alpha}-\dot{\phi}\right) .
$$

Now when we sum over $\alpha$ we can use the relations

$$
\sum\left|s_{\alpha}\right|^{2}=1, \sum \lambda_{\alpha}\left|s_{\alpha}\right|^{2}=\dot{\phi}
$$

to obtain

$$
F=\frac{1}{2}|\nabla \dot{\phi}|^{2}+\sum\left(\lambda_{\alpha}-\dot{\phi}\right)^{2}\left|s_{\alpha}\right|_{h_{0}}^{2}-|\nabla \dot{\phi}|^{2} .
$$

and so finally we see that

$$
\ddot{Z}=\int_{X} F d \mu_{h_{0}}
$$

(using again the condition $\sum\left|s_{\alpha}\right|^{2}=1$ ). Thus $\ddot{Z} \geq 0$ as required.

Corollary 2 If $H^{*} \in M$ is a balanced point then it is an absolute minimum of the function $\tilde{Z}=Z-\frac{V}{d} \log \operatorname{det}$ on $M$. 
To see this note that that $\log$ det is linear on geodesics, so $Z-\frac{V}{d} \log$ det is also convex on geodesics in $M$. Since any two points in $M$ can be joined by a geodesic any critical point is an absolute minumum.

(Just as Proposition 1 can be obtained from the group actions considered in [6] and general theory, so also one can see that the symmetrical result holds: the functional $\mathcal{L}$ is convex along geodesics in $\mathcal{K}$. However we will not go into this since the whole point of our proof is to avoid using the geodesics in $\mathcal{K}$.)

The other ingredient in the proof of Theorem 2 is the next Lemma.

Lemma 4 For any $h \in \mathcal{K}$ and $H \in M$ :

$$
\tilde{P}(h, H) \geq \tilde{P}(F S(H), H) .
$$

Choose an orthonormal basis $s_{\alpha}$ with respect to $H$ and let $h_{0}=F S(H)$, so $\sum\left|s_{\alpha}\right|_{h_{0}}^{2}=1$. Let $h=e^{\phi} h_{0}$ so $\sum\left|s_{\alpha}\right|_{h}^{2}=e^{\phi}$ and

$$
\sum\left\|s_{\alpha}\right\|_{\operatorname{Hilb}(h)}^{2}=\frac{d}{V} \int_{X} e^{\phi} d \mu_{h}
$$

Then, going back to the definitions, we find

$$
\tilde{P}(h, H)-\tilde{P}(F S(H), H)=\log \left(\frac{1}{V} \int_{X} e^{\phi} d \mu_{h}\right)-\frac{1}{V}\left(I(h)-I\left(h_{0}\right)\right)
$$

Now, by the convexity of the exponential function on $\mathbf{R}$,

$$
\log \left(\frac{1}{V} \int_{X} e^{\phi} d \mu_{h}\right) \geq \frac{1}{V} \int_{X} \phi d \mu_{h}
$$

and by Lemma 1

$$
\int_{X} \phi d \mu_{h} \geq I(h)-I\left(h_{0}\right)
$$

which completes the proof.

The proof of Theorem 2 is now in our hands. We have

$$
\tilde{P}(F S(H), H)=\log d+\frac{1}{V} \tilde{Z} .
$$

Suppose $\left(h^{*}, H^{*}\right)$ is a balanced pair as in (4). For any $(h, H)$,

$$
\tilde{P}(h, H) \geq \tilde{P}(F S(H), H) \geq \tilde{P}\left(F S\left(H^{*}\right), H^{*}\right)=\tilde{P}\left(h^{*}, H^{*}\right),
$$

by (17) and Corollary 2 and Lemma 4 . We also have

$$
\tilde{P}(h, \operatorname{Hilb}(h))=\log d+\frac{1}{d} \tilde{\mathcal{L}}(h),
$$


so taking $H=\operatorname{Hilb}(h)$ in $(18)$ we get

$$
\tilde{\mathcal{L}}(h) \geq \tilde{\mathcal{L}}\left(h^{*}\right)
$$

as required.

Notice that we also have the symmetrical result to Lemma 4:

Lemma 5 For any $h \in \mathcal{K}, H \in M$,

$$
\tilde{P}(h, H) \geq \tilde{P}(h, \operatorname{Hilb}(h)) .
$$

This is completely elementary, it just amounts to the standard inequality

$$
(\operatorname{det} Q)^{1 / d} \leq \frac{\operatorname{Tr}(Q)}{d}
$$

for $d \times d$ positive Hermitian matrices $Q$. Alternatively, it is a simple example of the "Kempf-Ness principle".

Using this Lemma we can obtain a slightly different result: whether or not a balanced point exists, the function $\tilde{\mathcal{L}}$ is bounded below on $\mathcal{K}$ if and only if the function $\tilde{Z}$ is bounded below on $M$.

\section{3}

We now move on to the second part of the proof of Theorem 1. We replace the line bundle $L$ by $L^{k}$ where $k$ is large and positive and we let $E_{k}$ be the complete linear system $H^{0}\left(X ; L^{k}\right)$. However we work with the same Kahler class $\mathcal{K}$. The whole discussion above goes through, with trivial changes. For $h \in \mathcal{K}$ we define functions $\rho_{k, h}$ on $X$ given by $\frac{\operatorname{dim} E_{k}}{V} \sum\left|s_{\alpha}\right|^{2}$ for a $\operatorname{Hilb}(h)$ orthonormal basis of $E_{k}$.

For a function $f$ on $X$ let us write

$$
[f]_{h}=f-\hat{f}
$$

where $\hat{f}$ is the average value of $f$ defined using the measure $d \mu_{h}$. We obtain functionals $\tilde{\mathcal{L}}_{k}$ on $\mathcal{K}$ with

$$
\delta \tilde{\mathcal{L}}_{k}=\int_{X}\left[\Delta^{\prime} \rho_{k, \phi}+k \rho_{k, \phi}\right]_{h} \delta \phi d \mu_{h}
$$

while the definition of the Mabuchi functional is

$$
\delta \mathcal{M}=\int_{X}[S]_{h} d \mu_{h}
$$


According to the results of Tian, Zelditch, Lu et al. (see the references cited in [6]: also the paper [16] and the new paper [4]),

$$
\left[\Delta^{\prime} \rho_{k, h}+k \rho_{k, h}\right]_{h} \sim \frac{1}{2 \pi} k^{n}[S]_{h},
$$

and the asymptotic relation holds uniformly over bounded sets of metrics (in a suitable sense), as stated in [6], Proposition 6. By integrating over paths in the space of metrics we deduce

Proposition 2 There are constants $\lambda_{k} \in \mathbf{R}$ such that

$$
\frac{2 \pi}{k^{n}} \tilde{\mathcal{L}}_{k}+\lambda_{k} \rightarrow \mathcal{M},
$$

as $k \rightarrow \infty$, uniformly over bounded subsets in $\mathcal{K}$.

Again, note that the Mabuchi functional is only defined up to an additive constant anyway.

Now suppose that there is a constant scalar curvature metric $\omega_{\infty}$ in the given Kahler class. This corresponds to some metric $h_{\infty} \in \mathcal{K}$. By the main result of [6] there is a sequence $h_{k} \in \mathcal{K}$, for large $k$, realising balanced metrics, and converging (in $C^{\infty}$ ) to $h_{\infty}$ as $k \rightarrow \infty$. Here we use the hypothesis on the automorphism group of $(X, L)$, required for the result in [6]. By Theorem 2, the $h_{k}$ are absolute minima of the functionals $\tilde{\mathcal{L}}_{k}^{\prime}=\frac{2 \pi}{k^{n}} \tilde{\mathcal{L}}_{k}+\lambda_{k}$. Now from the fact that the $\mathcal{L}_{k}^{\prime}$ converge to $\mathcal{M}$ uniformly over bounded sets of metrics it follows immediately that $h_{\infty}$ is an absolute minimum of the Mabuchi functional $\mathcal{M}$.

Remark. Notice that Lemma's 4 and 5 imply that the map $F S \circ$ Hilb : $M \rightarrow M$ decreases the functional $\tilde{Z}$. It follows easily (since $\tilde{Z}$ is proper) that iterates of this map converge to the minimiser. This gives an algorithm for finding balanced metrics numerically (when they exist), and hence an algorithm for finding constant scalar curvature metrics, when these exist.

\section{References}

[1] S. Bando and T. Mabuchi Uniqueness of Einstein-Kahler metrics modulo connected group actions In: Algebraic Geometry Advanced Studies in Pure Math. 11-40 1987

[2] X. Chen The space of Kahler metrics Jour. Differential Geometry 56 189-234 2000 
[3] X. Chen and G. Tian Geometry of Kahler metrics and holomorphic foliation by discs Preprint DG/0409433

[4] X. Dai, K. Liu and X. Ma On the asymptotic expansions of Bergman kernels Preprint DG/0404494

[5] S. Donaldson Symmetric spaces, Kahler geometry and Hamiltonian dynamics In: Northern California Symplectic Geometry Seminar (Eliashberg et al eds. ) Amer. Math. Soc 1999

[6] S.Donaldson Scalar curvature and projective embeddings, I Jour. Differential Geometry 59 479-522 2001

[7] S. Donaldson and P. Kronheimer The geometry of four-manifolds Oxford U.P. 1990

[8] G. Kempf and L. Ness The length of vectors in representation spaces In:Algebraic Geometry Lecture Notes in Math. Springer 732 233-243 1979

[9] T. Mabuchi K-energy maps integrating Futaki invariants Tohuku Math. Jour. 38 245-257 1986

[10] T. Mabuchi Some symplectic geometry on compact Kahler manifolds, I Osaka Jour. Math. 24 227-252 1987

[11] T. Mabuchi An energy-theoretic approach to the Hitchin-Kobayashi correspondence for manifolds, I To appear in Inventiones Math.

[12] T. Mabuchi An energy-theoretic approach to the Hitchin-Kobayashi correspondence for manifolds, II To appear in Jour. Differential Geometry.

[13] T. Mabuchi Stability of extremal Kahler manifolds To appear in Osaka Jour. Math.

[14] D. Phong and J. Sturm Scalar curvature, moment maps and the Deligns pairing Preprint DG/02009098

[15] D. Phong and J. Sturm Stability, energy functionals and Kahler-Einstein metrics Preprint DG/0203254

[16] W. Ruan Canonical coordinates and Bergman metrics Commun. Anal. Geom. 6 589-631 1998

[17] S. Semmes Complex Monge-Ampère and symplectic manifolds Amer. Jour. Math. 114 495-550 1992 
[18] S. Zhang Heights and reductions of semi-stable varieties Compositio Math. 104 77-105 1996 